This article may be downloaded for personal use only. Any other use requires prior permission of the author and AIP Publishing. The following article appeared in louchtchenko, D., \& Roy, P.-N. (2018). Ground states of linear rotor chains via the density matrix renormalization group. Journal of Chemical Physics, 148(13), 134115 and may be found at https://doi.org/ 10.1063/1.5024403 


\title{
Ground states of linear rotor chains via the density matrix renormalization group
}

\author{
Dmitri louchtchenko and Pierre-Nicholas Roya) \\ Department of Chemistry, University of Waterloo, Waterloo, Ontario N2L 3G1, Canada
}

(Received 31 January 2018; accepted 26 March 2018; published online 4 April 2018)

\begin{abstract}
In recent years, experimental techniques have enabled the creation of ultracold optical lattices of molecules and endofullerene peapod nanomolecular assemblies. It was previously suggested that the rotor model resulting from the placement of dipolar linear rotors in one-dimensional lattices at low temperature has a transition between ordered and disordered phases. We use the density matrix renormalization group (DMRG) to compute ground states of chains of up to 100 rotors and provide further evidence of the phase transition in the form of a diverging entanglement entropy. We also propose two methods and present some first steps toward rotational spectra of such molecular assemblies using DMRG. The present work showcases the power of DMRG in this new context of interacting molecular rotors and opens the door to the study of fundamental questions regarding criticality in systems with continuous degrees of freedom. Published by AIP Publishing. https://doi.org/10.1063/1.5024403
\end{abstract}

\section{INTRODUCTION}

The ability to produce endofullerenes by molecular surgery ${ }^{1}$ has resulted in a number of exciting results, both experimental ${ }^{2,3}$ and theoretical. ${ }^{4-9}$ The generation of carbon nanotube "peapods" has also recently been shown to be possible. ${ }^{10-15}$ The combination of these ideas leads to endofullerene peapods: carbon nanotubes which contain fullerene cages with atoms or molecules trapped inside. ${ }^{16-22}$

By treating these nanomolecular assemblies (NMAs) as fixed and rigid, we may study the motion of the atoms and molecules enclosed therein. The resulting model is similar in many respects to that obtained from placing ultracold particles in an optical lattice ${ }^{23,24}$ and has previously been studied in that context. ${ }^{25,26}$ Nevertheless, there are some fundamental differences: in an NMA, the imprisoned entities may not move between sites as they do in an optical lattice, so there cannot be double occupation of a site; the spacing between adjacent sites is much smaller in an NMA (on the order of $1 \mathrm{~nm}$ ) ${ }^{15}$ than in a typical optical lattice (on the order of $100 \mathrm{~nm}){ }^{23}$ and the carbon walls of the fullerene cages shield the interactions between the captive particles. ${ }^{3}$

For an endofullerene peapod NMA model as described above, one is in principle left with translational, vibrational, and rotational degrees of freedom for the confined particles. At very low temperatures, the translations and vibrations are restricted to their respective ground states, and only the rotational motion remains relevant, such that one can approximate the low-lying energy spectrum with an effective rotor Hamiltonian. In the following, we therefore focus on the rotational degrees of freedom of molecules arranged in a linear chain. Specifically, we choose dipolar linear rotor molecules (such as $\mathrm{HF}, \mathrm{LiCl}$, or $\mathrm{CsI}$ ) which interact pairwise through the

a)Electronic mail: pnroy@uwaterloo.ca (dimensionless) dipole-dipole potential

$$
V_{i j}\left(\mathbf{e}_{i}, \mathbf{e}_{j} ; \mathbf{r}_{i j}\right)=\mathbf{e}_{i} \cdot \mathbf{e}_{j}-3\left(\mathbf{e}_{i} \cdot \mathbf{r}_{i j}\right)\left(\mathbf{e}_{j} \cdot \mathbf{r}_{i j}\right),
$$

where $\mathbf{e}_{i}$ and $\mathbf{e}_{j}$ are unit vectors describing the orientation of two rotors and $\mathbf{r}_{i j}$ is the unit vector in the direction from one rotor to the other.

In this article, we propose a method for the calculation of ground state energies and wavefunctions for long one-dimensional systems of dipolar rotors using the density matrix renormalization group (DMRG). Originally introduced by White in $1992,{ }^{27}$ the approach of DMRG has proven fruitful in a number of applications ranging from condensed matter physics $^{28,29}$ to quantum chemistry. ${ }^{30-32}$ Although it has been extended to the study of two-dimensional systems, finite temperature systems, and real-time evolution, DMRG excels at finding ground states of strongly correlated one-dimensional systems. ${ }^{33}$

For small systems of this kind (up to around 10 rotors), sparse iterative methods for Hamiltonian diagonalization are sufficient to obtain a handful of low-lying eigenstates. ${ }^{34}$ As the systems grow, the size of the many-body basis increases exponentially and the problem quickly becomes intractable. Hence, we turn to DMRG in order to grow the rotor chain under study to 100 rotors, which is made feasible by the matrix product state (MPS) wavefunction ansatz inherent to DMRG. We accomplish this with the ITensor package, which allows us to efficiently formulate the Hamiltonian as a matrix product operator (MPO) and which contains an implementation of DMRG. ${ }^{35}$ While existing publications have also examined many-body quantum systems with dipole-dipole interactions using DMRG, ${ }^{25,36,37}$ due to their use of different geometries and focus on mapping to other model systems, they do not capture the full physics of interacting molecules under quantum rotation.

This paper is organized as follows: in Sec. II, we provide a brief introduction to matrix product states; in Sec. III 
we derive an expression for the dipole-dipole interaction potential in terms of angular momentum ladder operators; in Sec. IV, we show the results of our DMRG calculations for the rotor system; in Sec. V, we end with some concluding remarks.

\section{MATRIX PRODUCT STATES}

Despite originally being developed as a renormalization group technique, DMRG is now typically understood in terms of MPSs. ${ }^{29}$ When a wavefunction $|\psi\rangle$, which is an abstract element of a Hilbert space $\mathcal{H}=\mathcal{H}_{1} \otimes \mathcal{H}_{2} \otimes \cdots \otimes \mathcal{H}_{N}$, is represented in a finite (perhaps truncated) basis, it may be treated as a vector $C_{\mathbf{n}}=\langle\mathbf{n} \mid \psi\rangle$ of coefficients indexed by the multi-index $\mathbf{n}=\left(n_{1}, n_{2}, \ldots, n_{N}\right)$. Such a vector may always be expanded exactly into a product of matrices

$$
\langle\mathbf{n} \mid \psi\rangle=\underline{\mathbf{A}}^{(1), n_{1}} \underline{\mathbf{A}}^{(2), n_{2}} \cdots \underline{\mathbf{A}}^{(N), n_{N}} .
$$

Even though they do not necessarily correspond to physical sites on a lattice, the entities living in the $N$ one-body Hilbert spaces $\mathcal{H}_{k}$ are referred to as "sites." The collection $\left\{\underline{\mathbf{A}}^{(k)}\right\}$ of rank-3 tensors forms an MPS representation of $|\psi\rangle$, where $n_{k}$ is the physical index for the site and the two matrix indices (implied by the matrix multiplication) are bond indices with "bond dimension" $M_{k}$. [Each $\underline{\mathbf{A}}^{(1), n_{1}}$ is treated as a row vector and each $\underline{\mathbf{A}}^{(N), n_{N}}$ as a column vector, so that the product in Eq. (2) results in a scalar.] In practice, this expansion will not be made exact, as that leads to exponential scaling with system size; instead, the matrices are truncated to keep the bond dimension small.

We may arbitrarily group the sites into regions $A$ and $B$, and write the many-body Hilbert space as the product $\mathcal{H}=\mathcal{H}_{A} \otimes \mathcal{H}_{B}$. At the core of DMRG is the Schmidt decomposition

$$
|\psi\rangle=\sum_{i} \sqrt{\lambda_{i}}\left|\phi_{i}^{A}\right\rangle \otimes\left|\phi_{i}^{B}\right\rangle,
$$

where the $\lambda_{i}$ are non-negative real numbers, and $\left\{\left|\phi_{i}^{A}\right\rangle\right\}$ and $\left\{\left|\phi_{i}^{B}\right\rangle\right\}$ are orthonormal sets in $\mathcal{H}_{A}$ and $\mathcal{H}_{B}$, respectively. Truncation of the terms with the smallest coefficients results in an optimal approximation $|\tilde{\psi}\rangle$ in the sense of the 2-norm: $\||\psi\rangle-|\tilde{\psi}\rangle \|_{2}$ is minimized compared to other truncation schemes that retain the same number of terms. This truncation is typically performed so that

$$
\sum_{i} \lambda_{i} \leq \epsilon
$$

where the sum is over the discarded values and $\epsilon \ll 1$. Hence, this decomposition provides a systematic way to generate an efficient MPS. DMRG makes repeated use of this decomposition (along with iterative diagonalization, for example using the Lanczos algorithm) by "sweeping" the boundary between $A$ and $B$ from one end of the system to the other in order to obtain the ground state of a Hamiltonian.

The number of terms that remain in the sum dictates the bond dimension and has a direct impact on the difficulty of the calculation. The bond dimension also bounds the amount of entanglement that may be present across the corresponding boundary between the regions connected by the bond. The standard measure of bipartite entanglement for pure states is the von Neumann entropy

$$
S_{\mathrm{vN}}=-\operatorname{Tr}\left(\hat{\rho}_{A} \log \hat{\rho}_{A}\right),
$$

where $\hat{\rho}_{A}=\operatorname{Tr}_{B}|\psi\rangle\langle\psi|$ is the reduced density operator for region $A .{ }^{38}$ The entanglement entropy cannot exceed $\log M$, where $M$ is the dimension of the Hilbert space $\mathcal{H}_{A}$. When the boundary is at bond $k$, the dimension of the subspaces spanned by $\left\{\left|\phi_{i}^{A}\right\rangle\right\}$ and $\left\{\left|\phi_{i}^{B}\right\rangle\right\}$ is $M_{k}$, so the maximum possible von Neumann entropy is $S_{\mathrm{vN}}=\log M_{k}$. Conversely, the minimum bond dimension required to faithfully represent a state with entanglement entropy $S_{\mathrm{vN}}$ across bond $k$ is $M_{k}=e^{S_{\mathrm{VN}}}$. As these bounds are saturated only for maximally entangled states, the bond dimension will in general be larger than $e^{S_{\mathrm{vN}}}$.

In one spatial dimension and away from criticality, ground states of gapped Hamiltonians with short-range interactions are known to obey an entanglement area law. ${ }^{39}$ That is, the amount of entanglement between regions is proportional to the size of the boundary between them and is independent of the sizes of the regions themselves. Because a single boundary in one dimension must always have a constant size, the entanglement entropy of such states cannot depend on the size of the system and therefore the bond dimension will not increase when the system is made larger.

For long-range interactions, the picture is less clear. Recent work has shown that, under appropriate conditions, ground states of one-dimensional Hamiltonians with interactions that decay faster than $1 / r^{4}$ must satisfy an entanglement area law. ${ }^{40}$ For systems with even longer range interactions, such as the $1 / r^{3}$ considered in the present work, we are not aware of any proofs regarding entanglement area laws away from criticality. However, near a critical point, where conformal field theory takes over, the entanglement entropy scales logarithmically with system size in one dimension, ${ }^{41}$ which causes the bond dimension to grow linearly, increasing the computational cost of DMRG.

\section{DIPOLAR ROTORS}

For $N$ identical rotors with rotational constant $B$ and dipole moment $\mu$, the general Hamiltonian is

$$
\hat{H}=\frac{B}{\hbar^{2}} \sum_{i=1}^{N} \hat{\ell}_{i}^{2}+\frac{\mu^{2}}{4 \pi \epsilon_{0}} \sum_{i=2}^{N} \sum_{j=1}^{i-1} \frac{\hat{V}_{i j}}{r_{i j}^{3}},
$$

where $r_{i j}$ is the distance between rotors $i$ and $j$. Since a peapod NMA is inherently linear, without loss of generality, we may place the rotors along the $z$ axis and express the potential operator compactly as

$$
\hat{V}_{i j}^{(z)}=\hat{x}_{i} \hat{x}_{j}+\hat{y}_{i} \hat{y}_{j}-2 \hat{z}_{i} \hat{z}_{j}
$$

Because of the regular structure of a peapod NMA, we space the rotors evenly and write the Hamiltonian as

$$
\frac{\hat{H}}{B}=\sum_{i=1}^{N} \frac{\hat{\ell}_{i}^{2}}{\hbar^{2}}+\frac{1}{R^{3}} \sum_{i=2}^{N} \sum_{j=1}^{i-1} \frac{\hat{V}_{i j}^{(z)}}{(i-j)^{3}},
$$

where

$$
R=r\left(\frac{4 \pi \epsilon_{0} B}{\mu^{2}}\right)^{\frac{1}{3}}
$$


$r$ is the distance between adjacent rotors (the lattice spacing), and we have taken this opportunity to non-dimensionalize the Hamiltonian. Since all the physical properties appear only in $R$, the one-parameter form of the Hamiltonian allows us to explore the entire realm of physical realizations of this model by scanning a single parameter.

As the effective rotor Hamiltonian lacks the microscopic details describing the confinement of the rotors, it is general enough to apply to a variety of experimental situations in addition to NMAs, such as molecules trapped in a sufficiently deep optical lattice in which tunneling between minima is suppressed. The specifics of the physical configuration are encoded in $R$; for example, in an NMA, the dipole moment $\mu$ is screened by the fullerene cages, ${ }^{3}$ which causes an increase in $R$.

A natural one-body basis for this problem is that of the spherical harmonics $\left|\ell_{i} m_{i}\right\rangle$, in which the squared angular momentum operator $\hat{\ell}_{i}^{2}$ is diagonal

$$
\hat{\ell}_{i}^{2}\left|\ell_{i} m_{i}\right\rangle=\hbar^{2} \ell_{i}\left(\ell_{i}+1\right)\left|\ell_{i} m_{i}\right\rangle
$$

Although in principle this basis is infinite, in order to carry out any calculations, it will need to be truncated at a finite $\ell_{\max }$ so that it is large enough to accurately represent the quantities in question, but no larger.

Thanks to the form of the potential operator, the Hamiltonian in Eq. (8) conserves the total $\ell$ parity

$$
\ell_{\mathrm{p}} \equiv \sum_{i=1}^{N} \ell_{i} \quad(\bmod 2)
$$

and the total $m$ value

$$
m=\sum_{i=1}^{N} m_{i}
$$

In order to exploit the block-diagonal structure of the Hamiltonian in DMRG, we must make explicit use of these good quantum numbers. That is, we need to express the potential operator in terms of one-body operators that only change the quantum numbers $\ell_{\mathrm{p}}$ and $m$ by a definite amount, termed the "flux." This makes it possible to construct both the wavefunction MPS and Hamiltonian MPO as sparse objects, reducing the amount of storage required and significantly accelerating the calculation. ${ }^{29}$ Terms like $\hat{x}_{i} \hat{x}_{j}$ do not suffice because the position operators $\left(\hat{x}_{i}, \hat{y}_{i}, \hat{z}_{i}\right)$ do not have a well-defined flux. The action of one of these operators on a state with definite $\ell_{\mathrm{p}}$ and $m$ quantum numbers does not result in a state with definite $\ell_{\mathrm{p}}^{\prime}$ and $m^{\prime}$ values.

The ladder operators $\hat{\ell}_{i}^{ \pm}$and $\hat{m}_{i}^{ \pm}$, which raise and lower $\ell_{i}$ and $m_{i}$, are obvious candidates for building blocks, as their flux is immediately evident. The latter operators have the wellknown form

and they act as ${ }^{42}$

$$
\hat{m}_{i}^{ \pm}=\hat{\ell}_{i, x} \pm i \hat{\ell}_{i, y}
$$

$$
\hat{m}_{i}^{ \pm}\left|\ell_{i} m_{i}\right\rangle=\hbar \sqrt{\left(\ell_{i} \pm m_{i}+1\right)\left(\ell_{i} \mp m_{i}\right)}\left|\ell_{i}, m_{i} \pm 1\right\rangle .
$$

On the other hand, the ladder operators for $\ell_{i}$ do not appear to have been as deeply analyzed. There exist the definitions ${ }^{43}$

$$
\hbar \hat{R}_{i, z}=i\left(\hat{x}_{i} \hat{\ell}_{i, y}-\hat{y}_{i} \hat{\ell}_{i, x}\right)+\frac{\hat{z}_{i}}{2}\left(\hbar+\sqrt{4 \hat{\ell}_{i}^{2}+\hbar^{2}}\right)
$$

and

$$
\hbar \hat{Q}_{i, z}=i\left(\hat{x}_{i} \hat{\ell}_{i, y}-\hat{y}_{i} \hat{\ell}_{i, x}\right)+\frac{\hat{z}_{i}}{2}\left(\hbar-\sqrt{4 \hat{\ell}_{i}^{2}+\hbar^{2}}\right),
$$

but unfortunately $\hat{R}_{i, z}^{\dagger} \neq \hat{Q}_{i, z}$. We instead introduce the operators

$\hat{\ell}_{i}^{ \pm}=\frac{\hbar}{2} \hat{z}_{i}\left(1 \pm \frac{\hbar}{\sqrt{4 \hat{\ell}_{i}^{2}+\hbar^{2}}}\right) \pm i\left(\hat{x}_{i} \hat{\ell}_{i, y}-\hat{y}_{i} \hat{\ell}_{i, x}\right) \frac{\hbar}{\sqrt{4 \hat{\ell}_{i}^{2}+\hbar^{2}}}$

which are intimately related to $\hat{R}_{i, z}$ and $\hat{Q}_{i, z}$, but satisfy $\left(\hat{\ell}_{i}^{+}\right)^{\dagger}=\hat{\ell}_{i}^{-}$.

From this definition, it follows that

$$
\begin{aligned}
& \hat{x}_{i}=\frac{1}{2 \hbar^{2}}\left[\left(\hat{\ell}_{i}^{+}+\hat{\ell}_{i}^{-}\right),\left(\hat{m}_{i}^{+}-\hat{m}_{i}^{-}\right)\right], \\
& \hat{y}_{i}=\frac{1}{2 i \hbar^{2}}\left[\left(\hat{\ell}_{i}^{+}+\hat{\ell}_{i}^{-}\right),\left(\hat{m}_{i}^{+}+\hat{m}_{i}^{-}\right)\right],
\end{aligned}
$$

and

$$
\hat{z}_{i}=\frac{1}{\hbar}\left(\hat{\ell}_{i}^{+}+\hat{\ell}_{i}^{-}\right) .
$$

The clean and concise form of these expressions suggests that our choice of the ladder operators $\hat{\ell}_{i}^{ \pm}$is an appropriate one. The potential from Eq. (1) may then be written as

$$
\begin{aligned}
\hat{V}_{i j}= & \left(1-3 r_{i j, z}^{2}\right) \hat{B}_{i}^{0} \hat{B}_{j}^{0}-\frac{1}{4}\left[\left(1-3 r_{i j, z}^{2}\right) \hat{B}_{i}^{-} \hat{B}_{j}^{+}+3 r_{i j, \perp}^{2} \hat{B}_{i}^{-} \hat{B}_{j}^{-}\right. \\
& \left.+6 r_{i j, \perp} r_{i j, z}\left(\hat{B}_{i}^{-} \hat{B}_{j}^{0}+\hat{B}_{i}^{0} \hat{B}_{j}^{-}\right)+\text {h.c. }\right]
\end{aligned}
$$

where

$$
\begin{aligned}
& \hat{B}_{i}^{ \pm}= \pm \frac{1}{\hbar^{2}}\left(\left[\hat{\ell}_{i}^{+}, \hat{m}_{i}^{ \pm}\right]+\left[\hat{\ell}_{i}^{-}, \hat{m}_{i}^{ \pm}\right]\right), \\
& \hat{B}_{i}^{0}=\frac{1}{\hbar}\left(\hat{\ell}_{i}^{+}+\hat{\ell}_{i}^{-}\right),
\end{aligned}
$$

and

$$
r_{i j, \perp}=r_{i j, x}+i r_{i j, y} .
$$

The simplified form for rotors aligned along the $z$ axis is

$$
\hat{V}_{i j}^{(z)}=-2 \hat{B}_{i}^{0} \hat{B}_{j}^{0}+\frac{1}{2}\left[\hat{B}_{i}^{-} \hat{B}_{j}^{+}+\text {h.c. }\right] \text {. }
$$

When written in this form, the potential operator may be constructed as a sparse MPO.

\section{RESULTS}

In this section, we give some results for the ground state properties of the rotor system, as well as transition dipole moments (TDMs) for an excitation spectrum. The results were computed using DMRG with the MPS truncation parameter $\epsilon$ from Eq. (4) set to $10^{-10}$.

\section{A. Ground state properties}

The primary result of the DMRG routine is the ground state energy $E_{0}$. As these energies are expected to decrease with increasing system size $N$, we present them in the form of chemical potentials in Fig. 1. It is evident that a smaller value of $R$ (stronger interactions) requires a larger $\ell_{\max }$ (more basis states), as expected. For sufficiently large systems, we expect on physical grounds that the addition of a single 

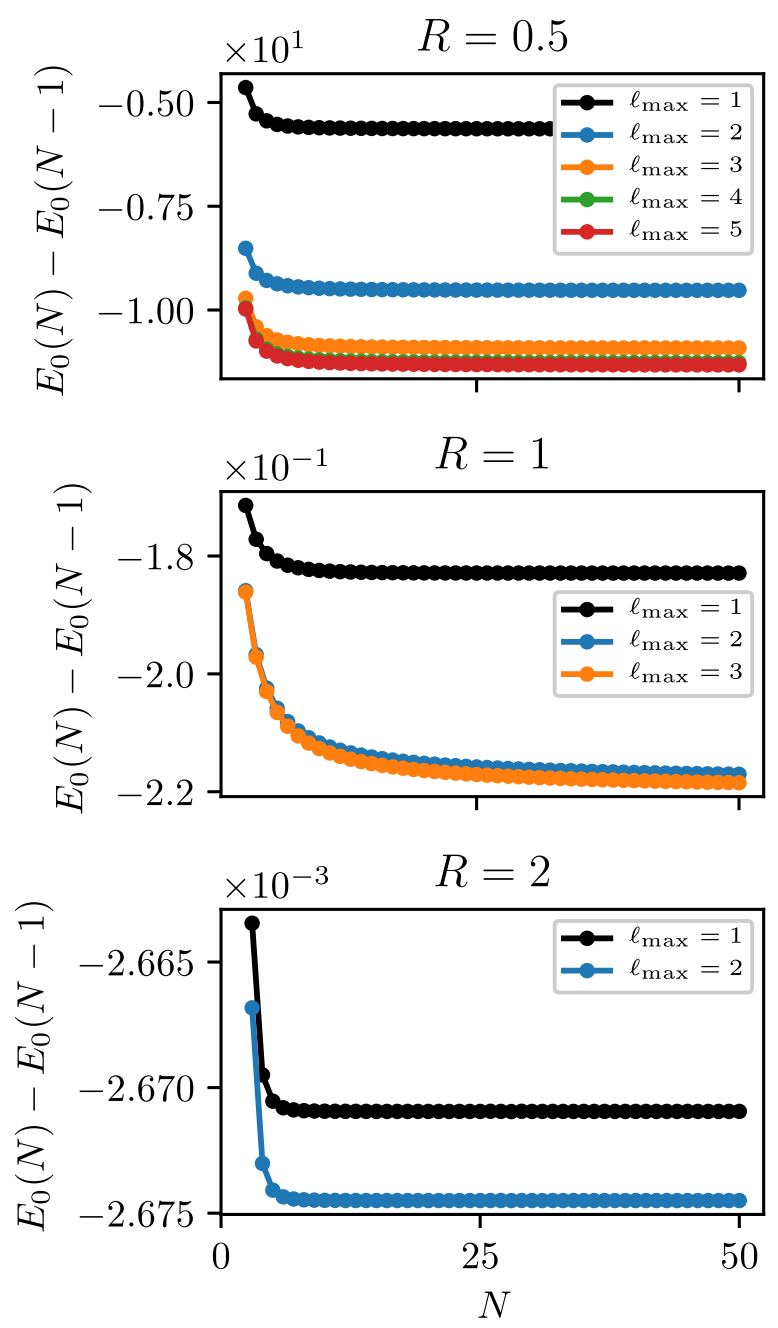

FIG. 1. Chemical potential of rotor chains of length $N$. Several curves with different $\ell_{\max }$ are shown to demonstrate the effect of basis truncation. The chemical potential at $R=1$ takes longer than the others to plateau.

particle will result in a constant decrease in the energy of the system, regardless of the system size. In other words, because a newly added rotor should only be substantially correlated with finitely many rotors on the end of the system, the chemical potential should tend to a constant in the large $N$ limit. For $R=0.5$ and $R=2$, this limit is reached by 25 rotors, but for $R=1$, the chemical potential continues to change even at 50 rotors, indicating longer ranged correlations.

The maximum bond dimension $M_{\max }$, shown in Fig. 2, is the largest bond dimension across the entire MPS, and it is indicative of the amount of long-range correlations in the state. That $M_{\max }$ plateaus quickly for $R=0.5$ and $R=2$ implies the presence of only short-range correlations, but the same cannot be said for $R=1$. The von Neumann entanglement entropy $S_{\mathrm{vN}}$ for the partitioning of the system into halves behaves similar to the bond dimension, as shown in Fig. 3. Indeed, we see what appears to be area law scaling at $R=0.5$ and $R=2$, as the entanglement does not change with system size once the system is large enough to make finite size effects negligible. At $R=1$, we instead notice what looks like an area law violation, possibly signifying a phase transition.
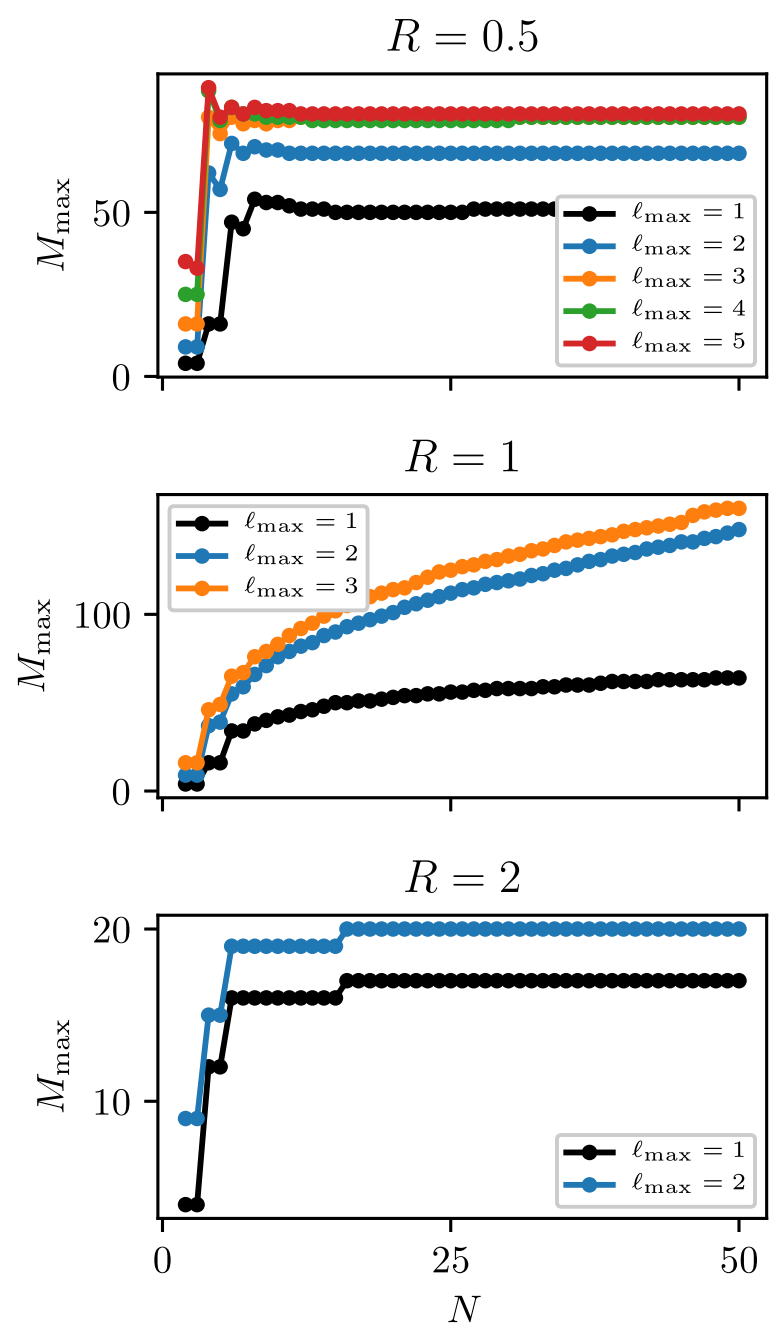

FIG. 2. Maximum MPS bond dimension for rotor chains of length $N$. Several curves with different $\ell_{\max }$ are shown to demonstrate the effect of basis truncation. The bond dimension at $R=1$ is larger than the others and not constant by $N=50$.

The quantum rotor model, which resembles the model used in the present article, but lacks the anisotropic term in Eq. (1), is known to have no ordered phase in one dimension and therefore no phase transition. ${ }^{44}$ In light of this, the observed anomalies at $R=1$ are peculiar, but it has been suggested that the breaking of rotational symmetry in the anisotropic model is responsible for a second-order phase transition between ordered and disordered phases. ${ }^{26}$ This is corroborated by the sudden change in both the expectation value of the orientational correlation operator

$$
\frac{2}{N(N-1)} \sum_{i=2}^{N} \sum_{j=1}^{i-1} \hat{\mathbf{e}}_{i} \cdot \hat{\mathbf{e}}_{j},
$$

and the von Neumann entanglement entropy $S_{\mathrm{vN}}$ near $R=1$, as demonstrated in Fig. 4. Of the two, it seems that the latter is a sharper indicator of the apparent phase transition. For larger system sizes, the entropy even peaks at $R=1$, clearly delineating the boundary between strongly and weakly interacting systems.

Though the maximum MPS bond dimension $M_{\max }$ is not a physical parameter, it contains valuable information 

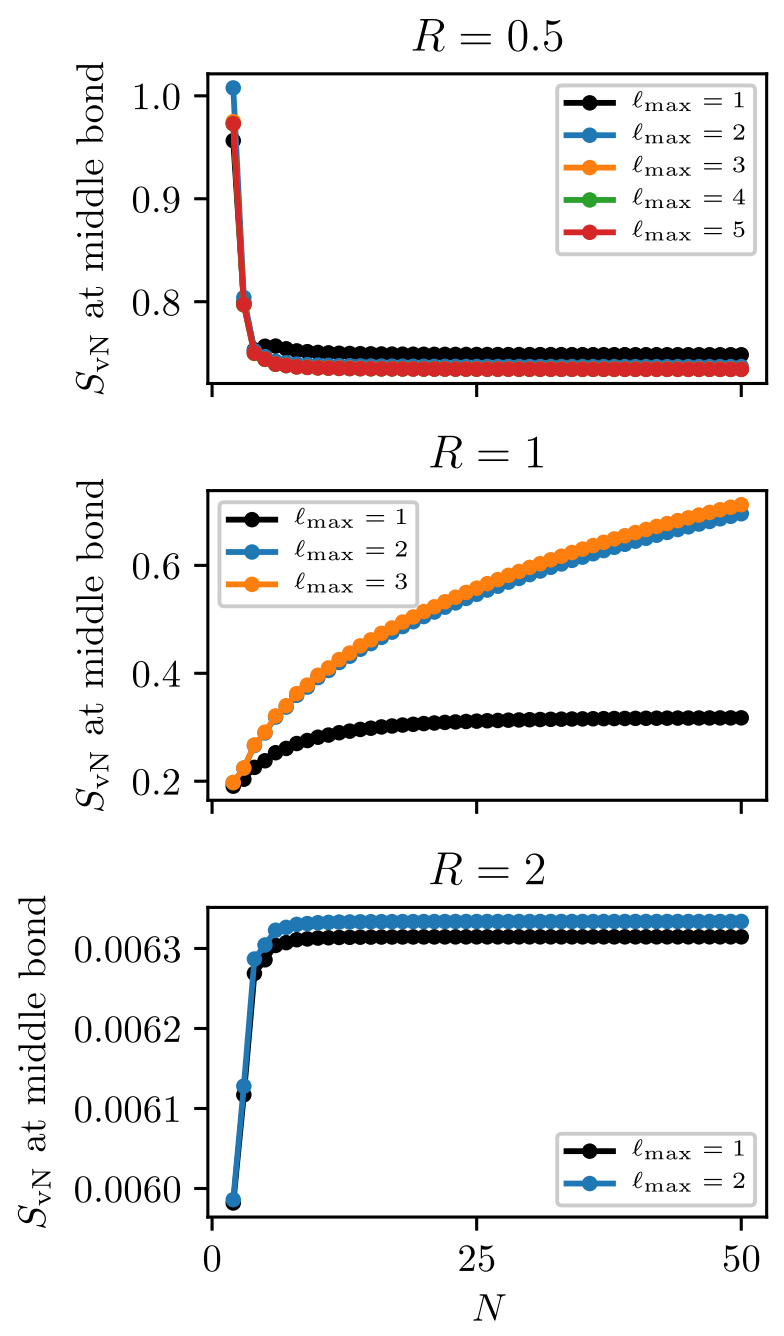

FIG. 3. von Neumann entanglement entropy of rotor chains of length $N$. Several curves with different $\ell_{\max }$ are shown to demonstrate the effect of basis truncation. The entropy at $R=1$ is still increasing by $N=50$.

about the effectiveness of DMRG for the system in question. As can be seen in Fig. 5, away from $R=1$, the maximum bond dimension converges very quickly, and we expect linear scaling of computational time. On the contrary, the peak at $R=1$ indicates that the scaling will not be as favorable, which is to be expected for DMRG near a second-order phase transition.

At such a phase transition, the spatial correlation length should also diverge. ${ }^{45}$ Thus, in the near future we plan to examine the behavior of the correlation length around $R=1$ to confirm the existence of the transition and identify the value of the critical parameter $R_{\mathrm{c}}$. We then hope to extract the central charge of the relevant conformal field theory for the critical system. $^{41}$

\section{B. Excitation spectrum}

Because the Hamiltonian in Eq. (8) is block-diagonal, we can also use DMRG to target the ground state of any symmetry block. The $\ell_{\mathrm{p}}=0, m=0$ block contains the ground state of the entire Hamiltonian, but the $\ell_{\mathrm{p}}=1, m=0$ and $\ell_{\mathrm{p}}=1$, $m= \pm 1$ blocks are also of interest, because their states $\left|n_{\ell_{\mathrm{p}}, m}\right\rangle$ are reachable from the ground state $\left|0_{\ell_{\mathrm{p}}=0, m=0}\right\rangle$ by application
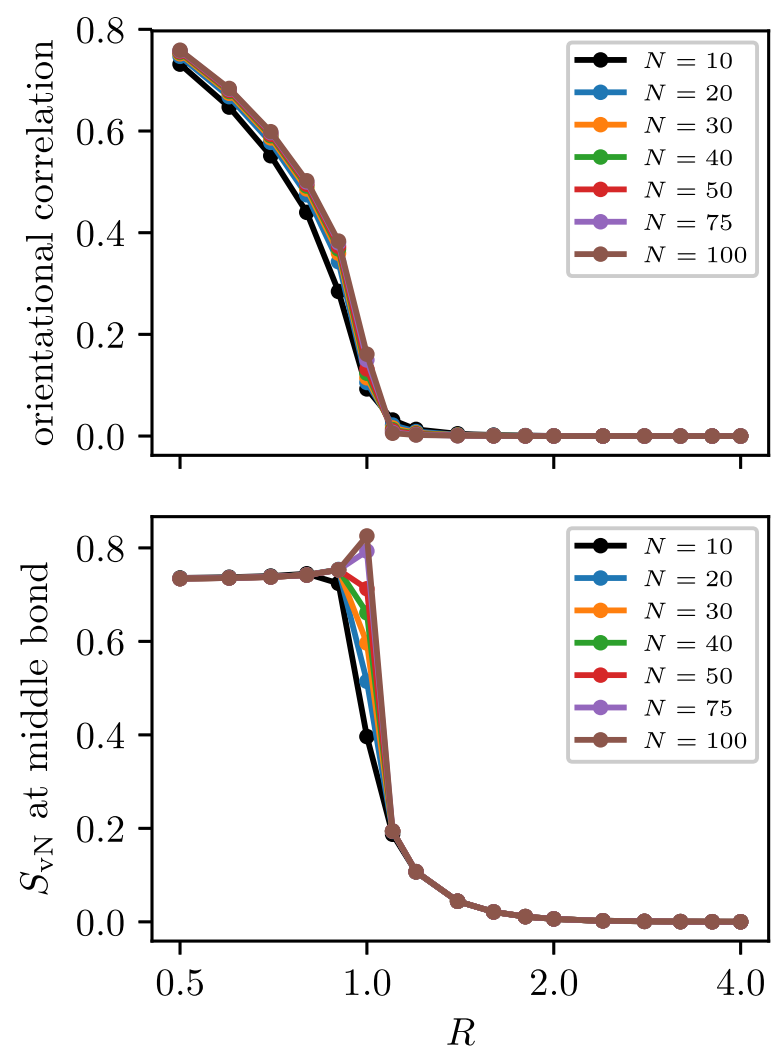

FIG. 4. Comparison of the orientational correlation (top panel) and the entanglement entropy (bottom panel) for rotor chains of size $N$ across the apparent phase transition around $R=1$.

of the $\hat{x}_{i}$ and $\hat{z}_{i}$ operators. That is, while most transition dipole moments (TDMs) are forbidden by symmetry, the moments

$$
\left|\left\langle n_{\ell_{\mathrm{p}}=1, m=0}\left|\frac{1}{N} \sum_{i=1}^{N} \hat{z}_{i}\right| 0_{\ell_{\mathrm{p}}=0, m=0}\right\rangle\right|^{2}
$$

and

$$
\left|\left\langle n_{\ell_{\mathrm{p}}=1, m= \pm 1}\left|\frac{1}{N} \sum_{i=1}^{N} \hat{x}_{i}\right| 0_{\ell_{\mathrm{p}}=0, m=0}\right\rangle\right|^{2}
$$

do not necessarily vanish.

Computed energy differences $\Delta E_{0}$ and TDMs for $R=2$ are listed in Table I. Despite this calculation providing only two peaks of a dipole excitation spectrum for each system size,

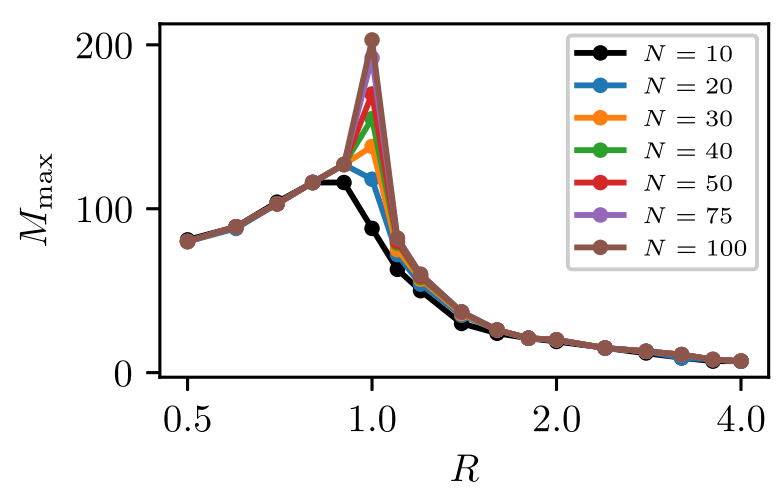

FIG. 5. Maximum MPS bond dimension for rotor chains of size $N$ across the apparent phase transition around $R=1$. There is a clear divergence with system size near $R=1$. 
TABLE I. Energy differences and transition dipole moments for rotor chains of length $N$ at $R=2$. Each value is computed between the ground state of the entire Hamiltonian and the ground state of the specified block at $\ell_{\max }=12$ and with 30 DMRG sweeps.

\begin{tabular}{lcccc}
\hline \hline$N$ & $\ell_{\mathrm{p}}$ & $m$ & $\Delta E_{0}$ & TDM \\
\hline 5 & 1 & 0 & 1.856 & $5.935 \times 10^{-2}$ \\
& 1 & \pm 1 & 1.950 & $3.117 \times 10^{-4}$ \\
\hline 10 & 1 & 0 & 1.843 & $2.296 \times 10^{-2}$ \\
& 1 & \pm 1 & 1.950 & $7.340 \times 10^{-5}$ \\
15 & 1 & 0 & 1.824 & $1.611 \times 10^{-2}$ \\
& 1 & \pm 1 & 1.948 & $3.504 \times 10^{-5}$ \\
\hline \hline
\end{tabular}

it lays the foundation for a series of more involved calculations which can reveal more information from the spectrum. We propose two complementary approaches for this. The first involves the direct calculation of excited states within the identified symmetry blocks. From these, more energy differences and TDMs can be computed, gradually populating a stick spectrum. The second requires time evolution of the ground state to obtain a correlation function, followed by a Fourier transform which yields a spectrum with finite resolution. Both approaches are presently possible using standard extensions to DMRG for excited states and real-time evolution. ${ }^{29}$

\section{CONCLUSIONS}

We have shown that DMRG can be applied to systems of dipolar linear rigid rotors which are pinned to the sites of a one-dimensional lattice. The method can be used to obtain ground state properties, such as the energy and von Neumann entanglement entropy. Excited states which are ground states of their respective symmetry blocks may also be reached by this method.

In order to perform the DMRG calculations efficiently, we have expressed the dipole-dipole potential in terms of angular momentum ladder operators $\hat{\ell}_{i}^{ \pm}$and $\hat{m}_{i}^{ \pm}$. These operators have a well-defined quantum number flux and they permit the use of an algorithm that conserves the total quantum numbers of the MPS. By making use of the symmetries of the Hamiltonian, which conserve $\ell_{\mathrm{p}}$ and $m$, we are able to apply our approach to a chain of 100 rotors.

Away from $R=1$, the computational time of the method scales linearly with system size and ground states of much longer chains with several hundred rotors may be computed relatively quickly. However, near $R=1$, the scaling is worse than linear and computing properties of larger systems becomes challenging. We attribute the favorable growth to area law scaling of the entanglement and its breakdown to a violation of the area law that is expected for a critical system.

An important extension to the above model is the addition of translational motion for the rotor molecules. ${ }^{6}$ This would take into account the interactions between the rotor molecule and the fullerene cage and would greatly enhance the applicability of the model to NMA experiments. The primary difficulty in implementing this change is the dynamical nature of the intermolecular separations: the classical parameter $R$ must be augmented by quantum mechanical operators which describe the deviations from the cage centers. Although it is not currently clear how such an implementation would look, it is likely to involve a model for the rotation-translation coupling which can be expressed in terms of raising and lowering operators for the site-local translational states.

\section{ACKNOWLEDGMENTS}

This research was supported by the Natural Sciences and Engineering Research Council of Canada (NSERC), the Ontario Ministry of Research and Innovation (MRI), the Canada Research Chair program, the Canada Foundation for Innovation (CFI), and the Canada First Research Excellence Fund (CFREF). We thank Martin Ganahl and Roger Melko for informative discussions.

${ }^{1}$ K. Komatsu, M. Murata, and Y. Murata, Science 307, 238 (2005).

${ }^{2}$ K. Kurotobi and Y. Murata, Science 333, 613 (2011).

${ }^{3}$ A. Krachmalnicoff, R. Bounds, S. Mamone, S. Alom, M. Concistrè, B. Meier, K. Kouřil, M. E. Light, M. R. Johnson, S. Rols et al., Nat. Chem. 8, 953 (2016).

${ }^{4}$ T. Rõ̃om, L. Peedu, M. Ge, D. Hüvonen, U. Nagel, S. Ye, M. Xu, Z. Bačić, S. Mamone, M. H. Levitt et al., Philos. Trans. R. Soc., A 371, 20110631 (2013).

${ }^{5}$ M. Xu, S. Ye, and Z. Bačić, J. Phys. Chem. Lett. 6, 3721 (2015).

${ }^{6}$ P. M. Felker and Z. Bačić, Chem. Phys. Lett. 683, 172 (2017).

${ }^{7}$ P. M. Felker and Z. Bačić, J. Chem. Phys. 146, 084303 (2017).

${ }^{8}$ P. M. Felker, V. Vlček, I. Hietanen, S. FitzGerald, D. Neuhauser, and Z. Bačić, Phys. Chem. Chem. Phys. 19, 31274 (2017).

${ }^{9}$ Y. N. Kalugina and P.-N. Roy, J. Chem. Phys. 147, 244303 (2017).

${ }^{10}$ B. W. Smith, M. Monthioux, and D. E. Luzzi, Nature 396, 323 (1998).

${ }^{11}$ B. W. Smith, M. Monthioux, and D. E. Luzzi, Chem. Phys. Lett. 315, 31 (1999).

${ }^{12}$ B. Burteaux, A. Claye, B. W. Smith, M. Monthioux, D. E. Luzzi, and J. E. Fischer, Chem. Phys. Lett. 310, 21 (1999).

${ }^{13}$ J. Sloan, R. E. Dunin-Borkowski, J. L. Hutchison, K. S. Coleman, V. C. Williams, J. B. Claridge, A. P. E. York, C. Xu, S. R. Bailey, G. Brown et al., Chem. Phys. Lett. 316, 191 (2000).

${ }^{14}$ M. Monthioux, Carbon 40, 1809 (2002).

${ }^{15}$ D. J. Hornbaker, S.-J. Kahng, S. Misra, B. W. Smith, A. T. Johnson, E. J. Mele, D. E. Luzzi, and A. Yazdani, Science 295, 828 (2002).

${ }^{16}$ K. Hirahara, K. Suenaga, S. Bandow, H. Kato, T. Okazaki, H. Shinohara, and S. Iijima, Phys. Rev. Lett. 85, 5384 (2000).

${ }^{17}$ B.-Y. Sun, T. Inoue, T. Shimada, T. Okazaki, T. Sugai, K. Suenaga, and H. Shinohara, J. Phys. Chem. B 108, 9011 (2004).

${ }^{18}$ H. Shiozawa, H. Rauf, T. Pichler, M. Knupfer, M. Kalbac, S. Yang, L. Dunsch, B. Büchner, D. Batchelor, and H. Kataura, Phys. Rev. B 73, 205411 (2006).

${ }^{19}$ R. Kitaura, H. Okimoto, H. Shinohara, T. Nakamura, and H. Osawa, Phys. Rev. B 76, 172409 (2007).

${ }^{20}$ R. J. Nicholls, K. Sader, J. H. Warner, S. R. Plant, K. Porfyrakis, P. D. Nellist, G. A. D. Briggs, and D. J. H. Cockayne, ACS Nano 4, 3943 (2010).

${ }^{21}$ W. L. Yang, Z. Y. Xu, H. Wei, M. Feng, and D. Suter, Phys. Rev. A 81, 032303 (2010).

${ }^{22}$ F. Fritz, R. Westerström, A. Kostanyan, C. Schlesier, J. Dreiser, B. Watts, L. Houben, M. Luysberg, S. M. Avdoshenko, A. A. Popov et al., Nanotechnology 28, 435703 (2017).

${ }^{23}$ A. Mazurenko, C. S. Chiu, G. Ji, M. F. Parsons, M. Kanász-Nagy, R. Schmidt, F. Grusdt, E. Demler, D. Greif, and M. Greiner, Nature 545, 462 (2017).

${ }^{24}$ M. E. Tai, A. Lukin, M. Rispoli, R. Schittko, T. Menke, D. Borgnia, P. M. Preiss, F. Grusdt, A. M. Kaufman, and M. Greiner, Nature 546, 519 (2017).

${ }^{25}$ A. V. Gorshkov, S. R. Manmana, G. Chen, J. Ye, E. Demler, M. D. Lukin, and A. M. Rey, Phys. Rev. Lett. 107, 115301 (2011).

${ }^{26}$ B. P. Abolins, R. E. Zillich, and K. B. Whaley, J. Low Temp. Phys. 165, 249 (2011).

${ }^{27}$ S. R. White, Phys. Rev. Lett. 69, 2863 (1992). 
${ }^{28}$ U. Schollwöck, Rev. Mod. Phys. 77, 259 (2005).

${ }^{29}$ U. Schollwöck, Ann. Phys. 326, 96 (2011).

${ }^{30}$ S. R. White and R. L. Martin, J. Chem. Phys. 110, 4127 (1999).

${ }^{31}$ G. K.-L. Chan and S. Sharma, Annu. Rev. Phys. Chem. 62, 465 (2011).

${ }^{32}$ C. Duperrouzel, P. Tecmer, K. Boguslawski, G. Barcza, Ö. Legeza, and P. W. Ayers, Chem. Phys. Lett. 621, 160 (2015).

${ }^{33}$ E. M. Stoudenmire and S. R. White, Annu. Rev. Condens. Matter Phys. 3, 111 (2012)

${ }^{34}$ T. Halverson, D. Iouchtchenko, and P.-N. Roy, J. Chem. Phys. 148, 074112 (2018).

${ }^{35}$ See http://itensor.org for ITensor library (version 2.1.1).

${ }^{36}$ J. Ruhman, E. G. Dalla Torre, S. D. Huber, and E. Altman, Phys. Rev. B 85, 125121 (2012)
${ }^{37}$ S. R. Manmana, E. M. Stoudenmire, K. R. A. Hazzard, A. M. Rey, and A. V. Gorshkov, Phys. Rev. B 87, 081106 (2013).

${ }^{38} \mathrm{M}$. Wilde, Quantum Information Theory (Cambridge University Press, 2013).

${ }^{39}$ M. B. Hastings, J. Stat. Mech.: Theory Exp. 2007, P08024.

${ }^{40}$ Z.-X. Gong, M. Foss-Feig, F. G. Brandão, and A. V. Gorshkov, Phys. Rev. Lett. 119, 050501 (2017).

${ }^{41}$ P. Calabrese and J. Cardy, J. Phys. A: Math. Theor. 42, 504005 (2009).

${ }^{42}$ L. E. Ballentine, Quantum Mechanics (Prentice-Hall, 1990).

${ }^{43}$ Q. H. Liu, D. M. Xun, and L. Shan, Int. J. Theor. Phys. 49, 2164 (2010).

${ }^{44}$ S. Sachdev, Quantum Phase Transitions, 2nd ed. (Cambridge University Press, 2011).

${ }^{45}$ J. Cardy, Scaling and Renormalization in Statistical Physics (Cambridge University Press, 1996). 